\title{
Real-World Outcome and Prognostic Factors of Pazopanib in Advanced Soft Tissue Sarcoma
}

\author{
Bader Alshamsan (iD ${ }^{1,2}$ \\ Ahmad Badran $\mathbb{D}^{1,3}$ \\ Aisha Alshibany' \\ Fatma Maraiki' \\ Mahmoud A Elshenawy ${ }^{1,4}$ \\ Tusneem Elhassan' \\ Jean Paul Atallah' \\ 'Medical Oncology, Oncology Center, \\ King Faisal Specialist Hospital and \\ Research Center, Riyadh, Saudi Arabia; \\ ${ }^{2}$ Department of Medicine, College of \\ Medicine, Qassim University, Buraydah, \\ Qassim, Saudi Arabia; ${ }^{3}$ Clinical Oncology \\ Department, Ain Shams University \\ Hospitals, Ain Shams, Cairo, Egypt; \\ ${ }^{4}$ Clinical Oncology, Faculty of Medicine - \\ Menoufia University, Shebeen El-Kom, \\ Shibin el Kom, Menoufia Governorate, \\ Egypt
}

Purpose: Pazopanib has been approved for treating soft tissue sarcomas (STS) after chemotherapy. We aimed to evaluate the prognostic factors, clinical outcomes, and tolerability of pazopanib in patients with STS.

Patients and Methods: Forty-five patients treated between June 2015 and August 2019 were reviewed. Clinical outcome was measured by assessing the disease control rate (DCR) using Response Evaluation Criteria in Solid Tumors (version 1.1). Progression-free survival (PFS) and overall survival (OS) were estimated using the Kaplan-Meier method. Adverse effects were assessed using the Common Terminology Criteria for Adverse Events (version 5.0).

Results: The median age of patients at diagnosis was 28 (interquartile range (IQR), 23-45) years. Pazopanib was used as the second-line treatment in $46.7 \%$ and the subsequent line in $53.3 \%$ of patients. The overall DCR was $55.6 \%$, and at 8 and 12 weeks, it was $52.3 \%$ and $35.5 \%$, respectively; the median duration of response was 7 (IQR: 2-18) months. Pazopanibinduced hypothyroidism was associated with DCR, with an odds ratio of 7 ( $95 \%$ confidence interval [95\% CI: 1.7-27.5], $\mathrm{p}<0.01$ ). The median PFS and OS were 4.1 (95\% CI: 0.85-7.42) and 12.4 months (95\% CI: 6.5-18.36), respectively. Hypothyroidism and response to pazopanib, better ECOG PS, histological subtypes desmoid tumor/aggressive fibromatosis (DT/ AF), and alveolar soft part sarcoma (ASPS) were favorable prognostic factors for PFS. Hypothyroidism and response to pazopanib were significant favorable factors for OS. There was no statistical difference in the OS between patients using pazopanib as the second-line therapy and those using it as the subsequent-line therapy.

Conclusion: Pazopanib is an effective treatment for STS. However, it showed variability in the clinical outcome in favor of ASPS and an outstanding response in the DT/AF subtype. Pazopanib-induced hypothyroidism is a good prognostic factor for disease control and is associated with prolonged PFS and OS.

Keywords: STS, a tyrosine kinase inhibitor, pazopanib, DT/AF, UPS, LMS, ASPS, Saudi Arabia

\section{Introduction}

Soft tissue sarcomas (STS) are a rare heterogeneous group of neoplasms that account for less than $1 \%$ of all solid cancers in adults. ${ }^{1,2}$ There are approximately 13,000 estimated new cases in the United States and 5000 estimated deaths annually. ${ }^{3}$ The age-standardized incidence rate (ASR) ranges between 1.5 and 3.0 per 100,000 people a year worldwide ${ }^{4,5}$ and 0.8 and 1 per 100,000 people in Saudi Arabia, respectively. ${ }^{6}$ Per the World Health Organization, there are more than 100 histological subtypes, with the most common subtypes being liposarcoma (20\%), leiomyosarcoma (LMS,14\%), undifferentiated pleomorphic sarcoma (UPS, 14\%), and
Correspondence: Bader Alshamsan Division of Medical Oncology, The

Ottawa Hospital Cancer Centre, Box 920

General Campus, 50I Smyth Road,

Ottawa, Ontario, KIH 8L6, Canada

Tel +I 6/37377700 Ext. 73467

Email bshmsan@qu.edu.sa 
gastrointestinal stromal tumors (GIST, 9\%). ${ }^{7}$ The treatment of STS requires a multidisciplinary team in specialized referral centers incorporating surgery, radiation therapy, and chemotherapy. The role of adjuvant chemotherapy is controversial, ${ }^{8}$ and nearly half of all patients experience recurrence. ${ }^{9}$ The current treatment option for metastatic STS is palliative chemotherapy, and single-agent doxorubicin has been the first-line treatment option for almost four decades. ${ }^{10}$ The combination of doxorubicin and ifosfamide increases response rates, but causes more adverse side effects with no survival advantage. ${ }^{11}$ The average response rate is between $22 \%$ and $53 \%$ depending on histological subtype and the patients' age. ${ }^{12}$ The median overall survival (OS) is around 1 year, which has not changed significantly over the past two decades, except the OS of patients with GIST. ${ }^{13}$ Second-line treatment options include eribulin, trabectedin, pazopanib, gemcitabine, docetaxel, vinorelbine, and dacarbazine as single agents, or a combination of gemcitabine and docetaxel or gemcitabine and dacarbazine. ${ }^{8}$ New drugs recently approved for STS treatment include pazopanib for non-liposarcoma, and avapritinib, repretinib, imatinib, sunitinib, and regorafenib for GIST. ${ }^{14}$ In general, after the third-line treatment, the best supportive care should be considered. ${ }^{15}$ Finally, tumor agnostic therapy is instituted with larotrectinib or entrectinib for tumors with fusions of the neurotrophic tyrosine receptor kinase (NTRK) genes and pembrolizumab for microsatellite instability sarcomas.

Pazopanib is a multi-tyrosine kinase inhibitor (TKI) of vascular endothelial growth factor receptors (VEGFR$1,-2$, and -3 ), platelet-derived growth factor (PDGFR- $\alpha$ and $-\beta$ ), fibroblast growth factor receptors (FGFR-1 and -3 ), and stem cell factor receptor (c-Kit), as well as other emerging targets. ${ }^{16}$ Pazopanib has been tested in Phase II trials, and the Phase III trial "PALETTE" has showed prolonged PFS with no significant effect on OS. ${ }^{14}$ Real-world data from eastern Asia indicate similar progression-free survival (PFS) advantages, highlight prognostic factors for clinical outcomes, and show toxicity profiles different from those of the PALETTE trial. ${ }^{17,18}$ Certainly, pazopanib has shown considerable evidence of the antitumor activity, and there is growing evidence of variations in tolerability to pazopanib between ethnicities and the presence of potential prognostic factors for the clinical outcome. Therefore, the objective of this retrospective study was to evaluate the clinical outcome, prognostic factors for disease control, PFS, and OS, as well as the tolerability to pazopanib in patients with STS in a real-world setting in Saudi Arabia and to compare the findings with published results.

\section{Materials and Methods}

\section{Patients and Methods}

This study was approved by the Research Advisory Council (RAC) at King Faisal Specialist Hospital and Research Centre (KFSHRC) (RAC number: 2191094). As the ethics committee did not mandate patients consent, a waiver of consent to use hospital records and databases was obtained from RAC at KFSHRC, Riyadh. All methods were carried out according to the Declaration of Helsinki and relevant guidelines and regulations.

The electronic medical records of patients with advanced STS who received pazopanib between June 2015 and August 2019 at KFSHRC, a tertiary hospital in Riyadh, were reviewed. The data included patient characteristics, Eastern Cooperative Oncology Group (ECOG) performance status (PS), baseline labs, staging, pathological features, and treatment lines, in addition to the best response at 8 and 12 weeks of pazopanib therapy, toxicity profile, time to progression, and status of the last follow-up, which was in February 2021. All patients underwent a baseline staging workup and subjected to response assessment every 2 months using the Revised Response Evaluation Criteria in Solid Tumors (RECIST version 1.1). ${ }^{19}$ The patients were followed-up based on the KFSH guidelines for clinical assessment at each visit, and their blood cell counts, renal and hepatic profiles, thyroid function, and treatment-related toxicities were evaluated. Treatment was continued until disease progression, unacceptable toxicity occurrence, or patient request to discontinue treatment. Clinical outcomes were measured by assessing disease control rate (DCR), PFS, and OS. DCR is the sum of CR, PR, and stable disease (SD) and assessed at best response, 8 and 12 weeks, The duration of response (DoR) is the time from confirmation of the first disease control (PR, CR, or SD) until progressive disease (PD) or death. PFS was defined as the time from the start of pazopanib until PD or death. OS was defined as the time from the start of pazopanib until death. The Common Terminology Criteria for Adverse Events (CTCAE) Version 5.0 was used as the toxicity grading system.

\section{Statistical Analysis}

Categorical values are described as frequency and continuous values are described as median with interquartile range (IQR). The association between various parameters 
and DCR to pazopanib was measured using the chi-square for categorical variables and Mann-Whitney $U$-test for continuous variables, and the significance of predictor was estimated by logistic regression. The Kaplan-Meier estimator was used to estimate the PFS and OS of pazopanib, and variables were compared using the Log rank test. Predictors were measured using Cox regression analysis. Statistical significance was set at $\mathrm{P}<0.05$. Statistical analysis was performed using Statistical Package for Social Sciences (SPSS) for Mac, Version 27 (IBM Corp, Armonk, NY, USA).

\section{Results}

\section{Patients Characteristics}

Forty-five Saudi patients with advanced STS were eligible for the analysis. The median age at diagnosis was 28 (IQR, 23-45) years. Patient and disease characteristics are presented in Table 1.

The most common histological types were UPS (26.7\%), synovial sarcoma (SS, 15.6\%), and leiomyosarcoma (LMS, 17\%). The majority of the patients (62\%) presented with metastatic disease at diagnosis, and the rest developed recurrence. The lung was the most common site of metastasis (55.6\%). Pazopanib was used as a second-line $(46.7 \%)$ or subsequent line $(53.3 \%)$ therapy.

\section{Disease Control Rate}

The overall DCR was $55.6 \%$, and the median DoR was 7 (IQR: 2-18) months. However, the DoR of eight patients was more than 1 year: 3 UPS, 3 desmoid tumor/aggressive fibromatosis (DT/AF), $1 \mathrm{SS}$, and 1 alveolar soft part sarcoma (ASPS). The detailed best response based on the RECIST criteria at 8 and 12 weeks is shown in Table 2.

The DCR for histological subtypes was as follows: LMS, 37.5\%; UPS, 58.3\%; SS, 57.1\%; ASPS, 75\%; DT/ AF, 100\%; and other sarcomas, 57.1\%. The chi-square test showed no significant difference $(\mathrm{P}=0.37)$. Logistic regression revealed an association between pazopanib-induced hypothyroidism and DCR (odds ratio (OR) 7, 95\% CI: $1.7-27.5 ; \mathrm{p}<0.01)$. The median duration for developing hypothyroidism was 2.03 (IQR, 0.93-5.24) months. There was no association between DCR and sex $(p=45)$, histological subtype $(\mathrm{p}=0.69)$, ECOG PS before pazopanib $(\mathrm{p}=0.16)$, pazopanib start dose $(\mathrm{p}=0.15)$, use of pazopanib in the second- or subsequent-line therapy $(\mathrm{p}=0.30)$, number of treatment lines before pazopanib $(p=0.40)$, metastasis at diagnosis $(p=0.42)$, recurrence type $(p=0.48)$, presence of lung metastasis $(p=0.60)$, liver metastasis $(\mathrm{p}=0.14)$, and pretreatment neutrophil-lymphocyte ratio (NLR) before pazopanib ( $\mathrm{p}=0.81)$. There was no association between DCR and weight loss $(p=0.30)$, hypertension $(p=0.18)$, or anemia $(p=0.15)$.

\section{Tolerability}

The starting dose was $800 \mathrm{mg}$ in 32 patients $(71.1 \%)$, $600 \mathrm{mg}$ in 8 patients $(17.8 \%$ ), and $400 \mathrm{mg}$ in 5 patients (11.1\%). The median duration of treatment was 4.1 (IQR 1.7-10.4) months. A dose reduction of $25 \%$ was required in 8 patients, $50 \%$ in 5 patients, and $>50 \%$ in 1 patient. The reason to stop pazopanib was disease progression in 33 patients $(73.3 \%)$, and 4 patients died during therapy, 3 patients stopped pazopanib due to toxicity, and 1 patient requested for discontinuation. Nine (20\%) patients received further treatment after pazopanib, and four patients remained on pazopanib until February 2021. The most common adverse effects were anemia, weight loss, hypertension, and hypothyroidism - the details of adverse effects are illustrated in Table 3.

\section{Survival Analysis}

The median duration of follow-up was 12.4 (IQR: 3.521.1) months. The median PFS was 4.1 (95\% CI: $0.85-$ 7.42) months, and the median OS was 12.4 (95\% CI: $6.5-$ 18.36) months (Figure 1).

The Log rank test revealed an association between the median PFS and ECOG PS, response to pazopanib, and presence of hypothyroidism (Figure $2 \mathrm{~A}-\mathrm{C}$ ). The median PFS based on ECOG PS before starting pazopanib was 5.1, 5, and 1.2 months for ECOG PS 0/I, II, and III, respectively $(\mathrm{P}=0.01)$. The median $\mathrm{PFS}$ in patients with disease response to pazopanib vs no response was 7.9 (95\% CI: 4.40-11.45) vs 1.7 (95\% CI: $1.54-1.95)$ months $(\mathrm{p}<0.01)$, respectively. The median PFS in patients who developed hypothyroidism vs no hypothyroidism was 8.3 (95\% CI: 4.1-12.6) vs 2 (95\% CI: 1.3-2.7) months, $\mathrm{P}<0.001$, respectively.

The median PFS was higher in patients who received the standard dose, $800 \mathrm{mg}$ vs $\leq 600 \mathrm{mg}, 5$ months $(95 \%$ CI:0.50-19.39) vs 3 months (95\% CI: 1.28-4.72); however, this was not statistically significant $(p=0.23)$. Furthermore, hypertension was associated with clinical differences in median PFS of 5 months vs 2.9 months $(p=0.09)$. The PFS was not associated with sex $(p=0.3)$, and that with the use of pazopanib as the second-line treatment was 5.1 (95\% CI: 1.1-12.4) vs subsequent 
Table I Patients and Disease Characteristics $(n=45)$

\begin{tabular}{|c|c|}
\hline Characteristics & Number (\%) \\
\hline Age at diagnosis Median (IQR) & $28(23.5-45)$ \\
\hline \multicolumn{2}{|l|}{ Sex } \\
\hline Male & $23(51.1)$ \\
\hline Female & $22(48.9)$ \\
\hline \multicolumn{2}{|l|}{ Histology } \\
\hline Undifferentiated pleomorphic sarcoma (UPS) & $12(26.7)$ \\
\hline Synovial sarcoma (SS) & $7(15.6)$ \\
\hline Leiomyosarcoma (LMS) & $8(17)$ \\
\hline Alveolar soft part sarcoma (ASPS) & $4(8.9)$ \\
\hline Desmoid tumor/Aggressive fibromatosis (DT/AF) & $4(8.9)$ \\
\hline Epithelioid sarcoma & $3(6.7)$ \\
\hline Malignant peripheral nerve sheath tumor (MPNST) & $\mathrm{I}(2.2)$ \\
\hline Desmoplastic small round cell tumor (DSRCT) & $\mathrm{I}(2.2)$ \\
\hline Others & $5(I I . I)$ \\
\hline Metastasis at diagnosis & $28(62)$ \\
\hline \multicolumn{2}{|l|}{ Metastatic Sites } \\
\hline Lung & $25(55.6)$ \\
\hline Liver & $6(13.3)$ \\
\hline Brain & $3(6.6)$ \\
\hline Bone & $2(4.4)$ \\
\hline \multicolumn{2}{|l|}{ Type of recurrence } \\
\hline Local & $3(6.7)$ \\
\hline Distant & $26(57.8)$ \\
\hline Both & $16(35.6)$ \\
\hline \multicolumn{2}{|l|}{ Prior chemotherapy } \\
\hline Anthracycline & $30(66.6)$ \\
\hline Ifosfamide & $24(53.3)$ \\
\hline Gemcitabine & $13(28.8)$ \\
\hline Docetaxel & II (24.4\%) \\
\hline The median number of chemotherapy before & 2 (IQR: I-4) \\
\hline Pazopanib & \\
\hline Surgery & $30(66.7)$ \\
\hline \multicolumn{2}{|l|}{ Pazopanib } \\
\hline Second line & $21(46.7)$ \\
\hline Subsequent line & $24(53.3)$ \\
\hline \multicolumn{2}{|l|}{ ECOG PS before starting Pazopanib } \\
\hline $0 / 1$ & $34(75.6)$ \\
\hline 2 & $8(17.8)$ \\
\hline 3 & $3(6.7)$ \\
\hline
\end{tabular}

Abbreviation: ECOG PS, Eastern Cooperative Oncology Group Performance Status.

lines was 3 (95\% CI: $0.5-6.7)$ months $(p=0.48)$. There were no differences in the median PFS with metastasis at
Table 2 Best Response of Pazopanib at 8 and 12 Weeks

\begin{tabular}{|l|l|l|l|}
\hline $\begin{array}{l}\text { Best } \\
\text { Response }\end{array}$ & $\begin{array}{l}\text { Best Response } \\
(\mathbf{n = 4 5 )}\end{array}$ & $\begin{array}{l}\text { At 8 Weeks } \\
(\mathbf{n = 4 2 )}\end{array}$ & $\begin{array}{l}\text { At I2 Weeks } \\
(\mathbf{n}=45)\end{array}$ \\
\hline CR & 0 & 0 & 0 \\
PR & 9 & 7 & 6 \\
SD & 16 & 15 & 10 \\
PD & 20 & 20 & 29 \\
DCR (CR & $55.6 \%$ & $52.3 \%$ & $35.5 \%$ \\
$+P R+S D)$ & & & \\
\hline
\end{tabular}

Abbreviations: $C R$, Complete response; PR, Partial response; SD, Stable disease; PD, Progressive disease; DCR, Disease control rate.

diagnosis $(\mathrm{p}=0.07)$, type of recurrence $(\mathrm{p}=0.059)$, sites of metastasis $(\mathrm{p}=0.2)$, pretreatment NLR $(\mathrm{p}=0.56)$, presence of weight loss $(\mathrm{p}=0.99)$, or anemia $(\mathrm{p}=0.40)$. The multivariate Cox regression remained significant for hypothyroidism (hazard ratio (HR) 0.40, 95\% CI: 0.18-0.90), $\mathrm{p}=0.02$ and disease response to pazopanib (HR 0.22, 95\% CI: $0.10-0.51), \mathrm{p}<0.001$.

The factors associated with the median OS were response to pazopanib 22 (95\% CI: 16-27.9) vs 4 (95\% CI: 1.7-6.2) months $(\mathrm{p}<0.001)$, hypothyroidism not reached vs 5 months (95\% CI: 3.3-6.6 months) $(\mathrm{p}<0.001)$, and ECOG PS before pazopanib, 17, 8, 2 months for PS 0/I, II, and III, respectively $(\mathrm{p}=0.04)$. There was no association with sex $(p=0.16)$, metastasis at diagnosis $(\mathrm{p}=0.053)$, sites of metastasis $(\mathrm{p}=0.15)$, order of pazopanib $(\mathrm{p}=0.79)$, presence of weight loss $(\mathrm{p}=0.15)$, anemia $(\mathrm{p}=0.38)$, hypertension $(\mathrm{p}=0.09)$, or pretreatment NLR $(\mathrm{p}=0.18)$. The patients who started with $800 \mathrm{mg}$ had higher median OS than those who started with $\leq 600 \mathrm{mg}, 14.1$ (95\% CI: 0.93-27.39) vs 8.6 (95\% CI: 1.11-16.14) months, respectively. However no statistical significance was observed $(\mathrm{p}=0.32)$.

The multivariate Cox regression analysis only remained significant for hypothyroidism (HR 0.13, 95\% CI: 0.04 $0.38, \mathrm{p}<0.001$ ) (Figure $3 \mathrm{~A}$ ), and disease response to pazopanib (HR 0.32, 95\% CI: 0.14-0.73, $\mathrm{p}=0.007$ ) (Figure 3B).

The median $(95 \% \mathrm{CI})$ PFS for histological subtypes was as follows: LMS 1.75 (1.4-2), UPS 3 (0.5-9.4), SS 5.1 (4.75.6) ASPS 7.9 (1.1-18.11), DT/AF 31 (7.5-NR), and other sarcomas 2 (1-2.9), $(\mathrm{P}=0.01)$ (Figure 4A). The median (95\% CI) OS for histological subtypes was as follows: LMS, 9.5 (2.3-20.3); UPS, 5.6 (0.4-14); SS, 12.4 (7.4-17.3); ASPS, 14.1 (1.9-26.3); and other sarcomas 3.1, (0.86-7.6) months, $(\mathrm{p}=0.46)$ (Figure 4B). All DT/AF cases were censored, with no deaths, and two of them still received pazopanib. 
Table 3 Pazopanib-Related Adverse Effects

\begin{tabular}{|c|c|c|c|c|}
\hline Adverse event & Overall Number (\%) & Grade I/II Number (\%) & Grade III/IV Number (\%) & Grade V Number (\%) \\
\hline Hypertension & $23(5 \mathrm{I} .1)$ & $21(46.7)$ & $2(4.4)$ & \\
\hline Fatigue & $9(20)$ & $7(15.5)$ & $\mathrm{I}(2.2)$ & $\mathrm{I}(2.2)$ \\
\hline Weight loss & $24(53.3)$ & $23(5 \mathrm{I} .1)$ & $\mathrm{I}(2.2)$ & \\
\hline \multicolumn{5}{|l|}{ Hematological } \\
\hline Thrombocytopenia & $15(33.3)$ & $13(28.9)$ & $2(4.4)$ & \\
\hline Anemia & $28(62.2)$ & $26(57.8)$ & $2(4.4)$ & \\
\hline Neutropenia & $12(26.6)$ & $10(22.2)$ & $2(4.4)$ & \\
\hline Transaminases & $14(31.1)$ & $13(28.9)$ & & $\mathrm{I}(2.2)$ \\
\hline \multicolumn{5}{|l|}{ Electrolytes } \\
\hline Hypomagnesemia & $7(15.5)$ & $7(15.5)$ & & \\
\hline Hypophosphatemia & $8(17.8)$ & $8(17.8)$ & & \\
\hline Hypokalemia & $6(13.3)$ & $6(13.3)$ & & \\
\hline Hypernatremia & $5(\mathrm{II} . \mathrm{I})$ & $5(11.1)$ & & \\
\hline Hypothyroidism & $18(40)$ & $18(40)$ & & \\
\hline ILD & I (2.2) & & & $\mathrm{I}(2.2)$ \\
\hline
\end{tabular}

Abbreviation: ILD, Interstitial lung disease.

\section{Discussion}

This is a cohort of patients from Saudi Arabia with metastatic STS who received pazopanib treatment. The most common histological subtypes were UPS (26.7\%), SS (15.6\%), and LMS (17\%). The overall DCR was 55.6\%, and at 8 and 12 weeks, it was $52.3 \%$ and $35.5 \%$, respectively, across all subtypes, with the median DoR of 3 months. The median PFS and OS were 4.1 and 12.4 months, respectively. There was no statistically significant difference in DCR for histological subtypes; however, there was a clear trend of better DCR with DT/AF and ASPS subtypes. Moreover, there was a significant difference in the median PFS between the histological subtypes in favor of DT/AF and ASPS. The most common adverse effects were anemia, weight loss, hypertension, and hypothyroidism, with an infrequent incidence of grade 3-4 toxicities. The presence of hypothyroidism and

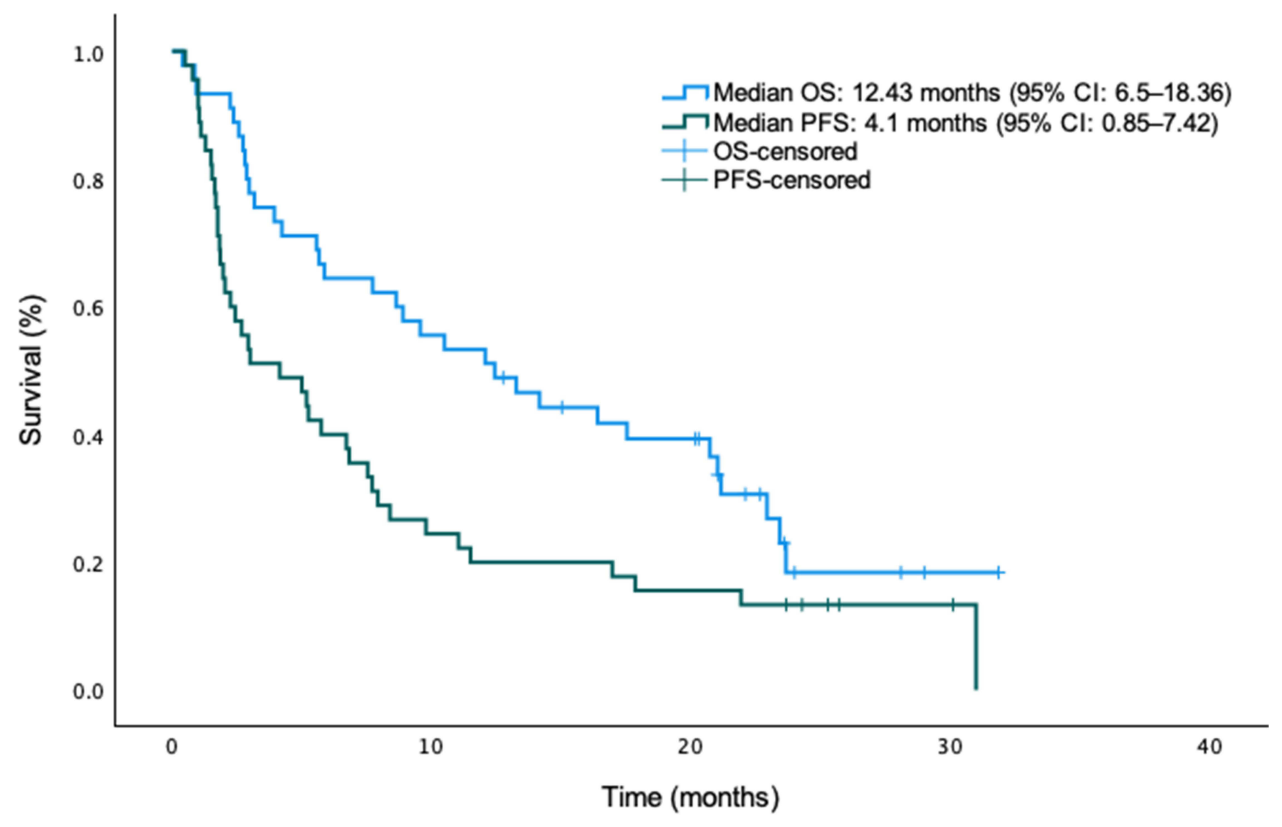

Figure I Kaplan-Meier curves for progression-free survival and overall survival of pazopanib in soft tissue sarcoma. 


\section{A}

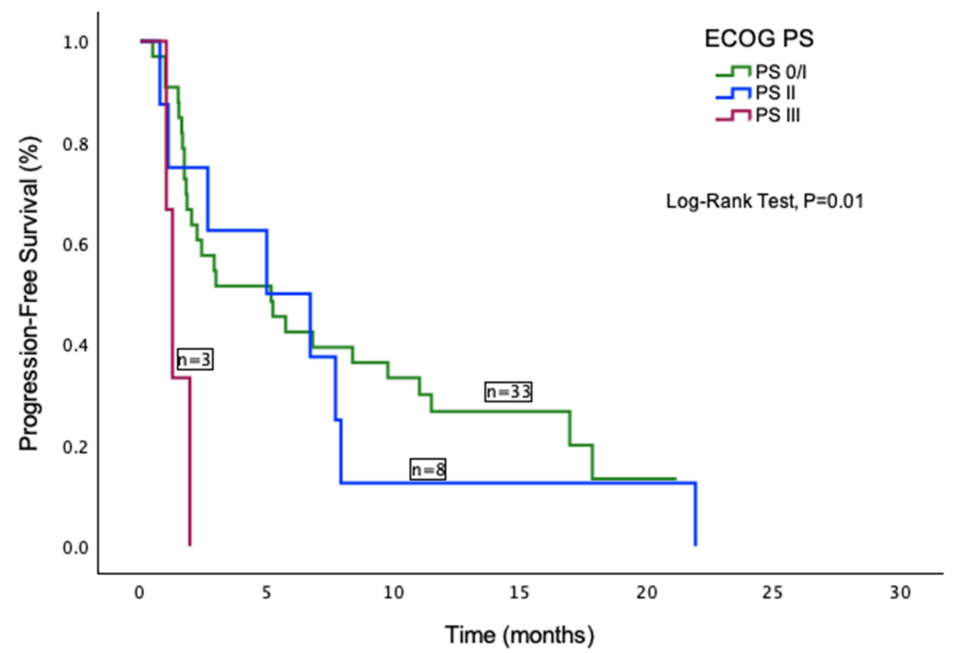

B

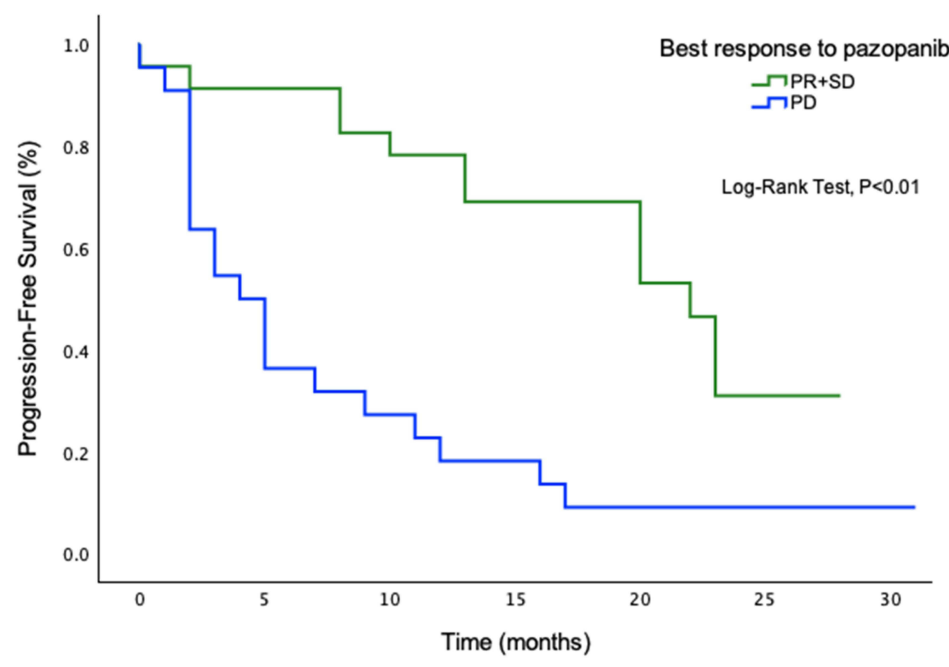

C

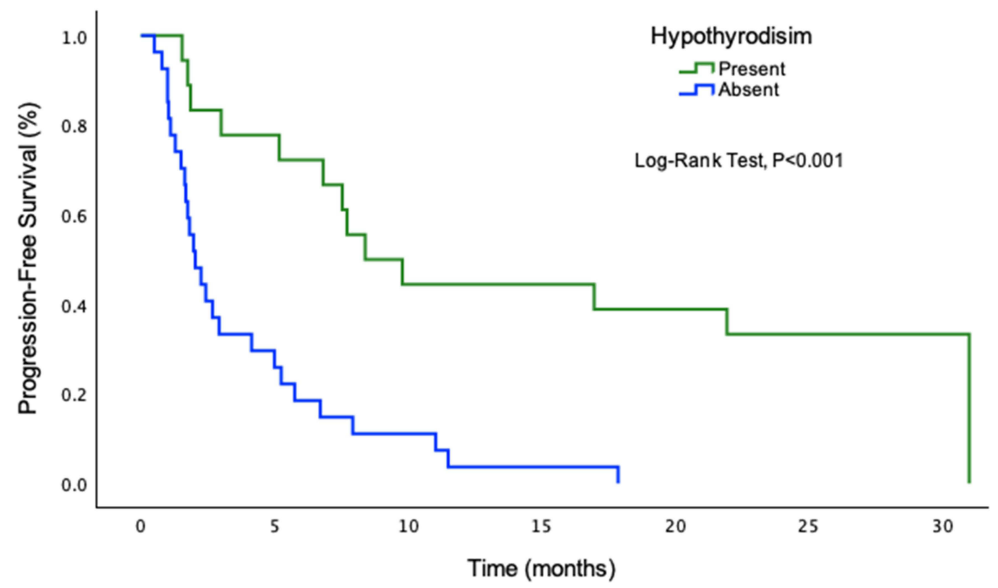

Figure 2 Kaplan-Meier curves for progression-free survival based on ECOG PS (A), best response to pazopanib (B), and hypothyroidism (C). 


\section{A}

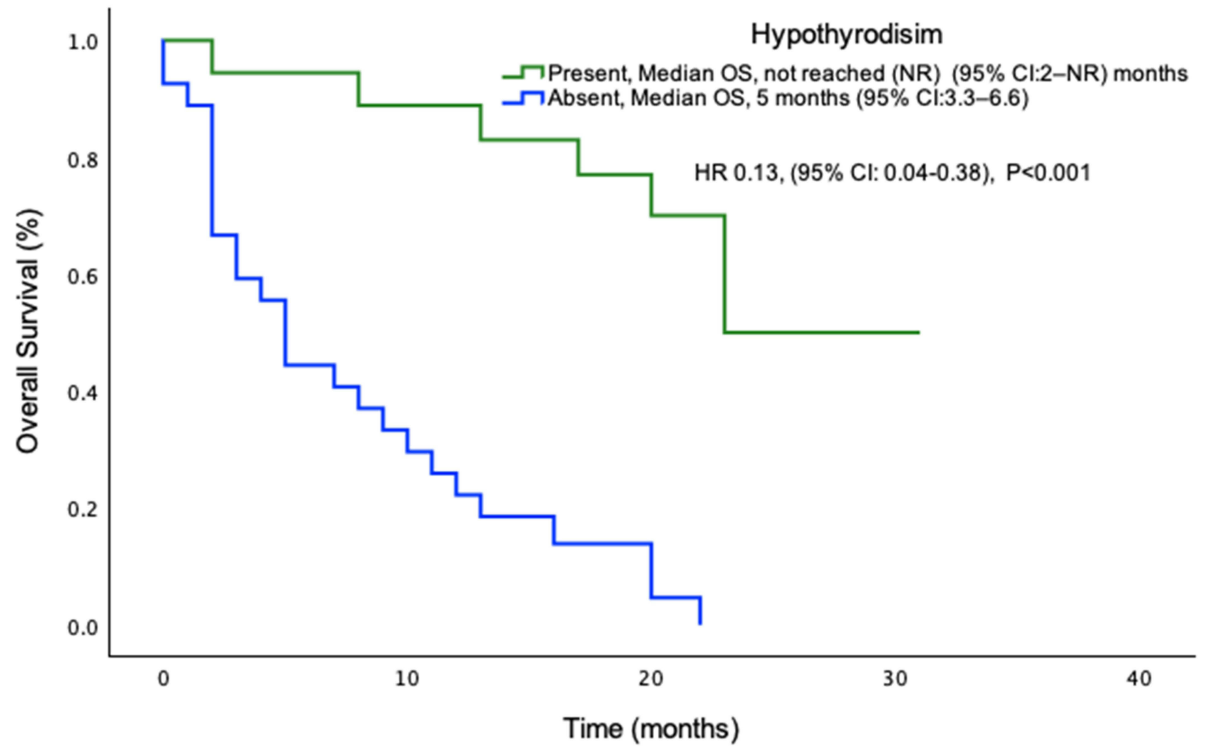

B

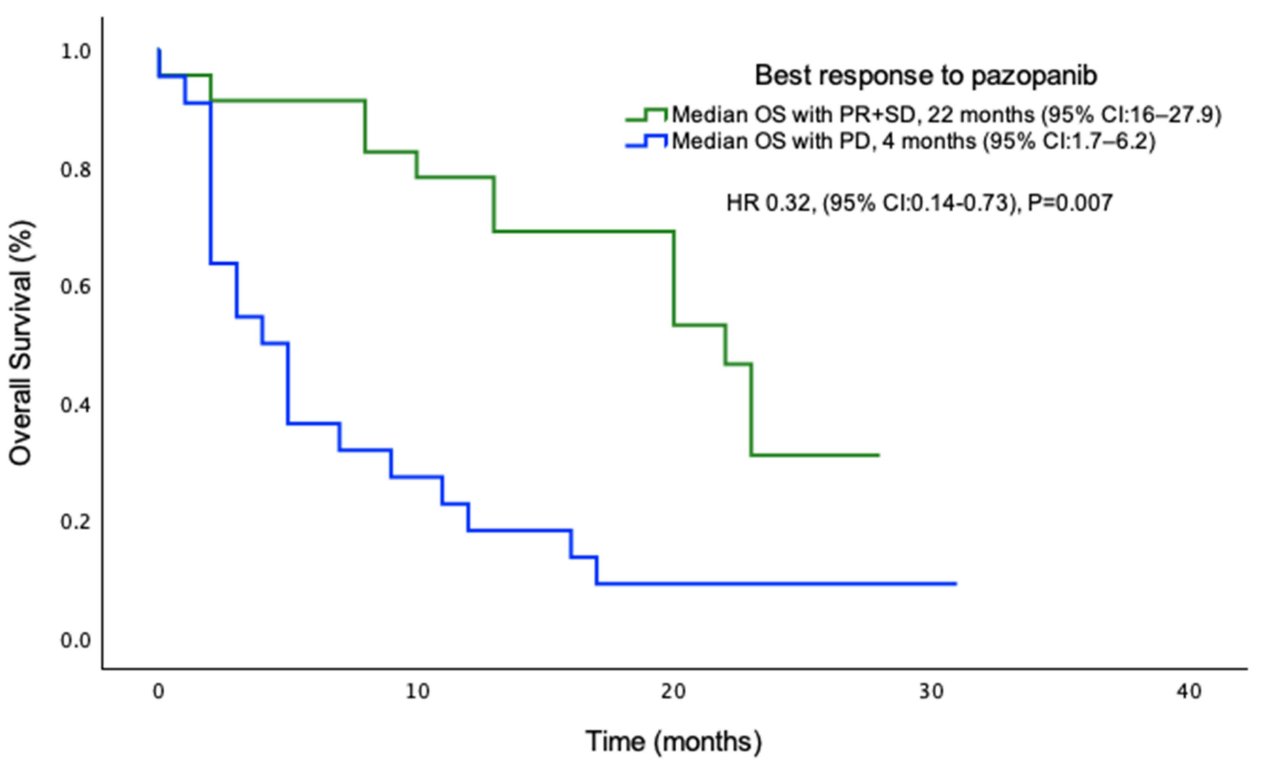

Figure 3 Kaplan-Meier curves for overall survival based on hypothyroidism (A) and best response to pazopanib (B).

response to pazopanib and better ECOG PS have been associated with prolonged PFS and OS; however, only hypothyroidism and response to pazopanib remained significant in multivariate Cox regression. Moreover, hypothyroidism is a favorable prognostic factor for the disease response.

The better DCR in DT/AF and ASPS could be related to the relatively less aggressive nature of these histological subtypes than the other histological subtypes. However, the promising effectiveness of pazopanib in DT/AF was highlighted for the first time in a case report, which reported that $\mathrm{CR}$ was achieved at the year follow-up.
Therefore, 2 and 8 cases have been reported separately. The DCR was $100 \%$ in all patients, and none of them showed progression with pazopanib; the median duration of treatment was 1 year. ${ }^{20-22}$ Moreover, a recent phase two study showed higher PFS in patients on pazopanib vs methotrexate and vinblastine in a 6-month assessment. ${ }^{23}$ A phase II trial in Japan reported disease control of $80 \%$ at 12 weeks in 5 patients with ASPS. ${ }^{24}$

In this study, we observed comparable clinical outcomes and tolerability with those of the phase III PALETTE trial $^{14}$ and real-world data. ${ }^{17,25-29}$ In the 


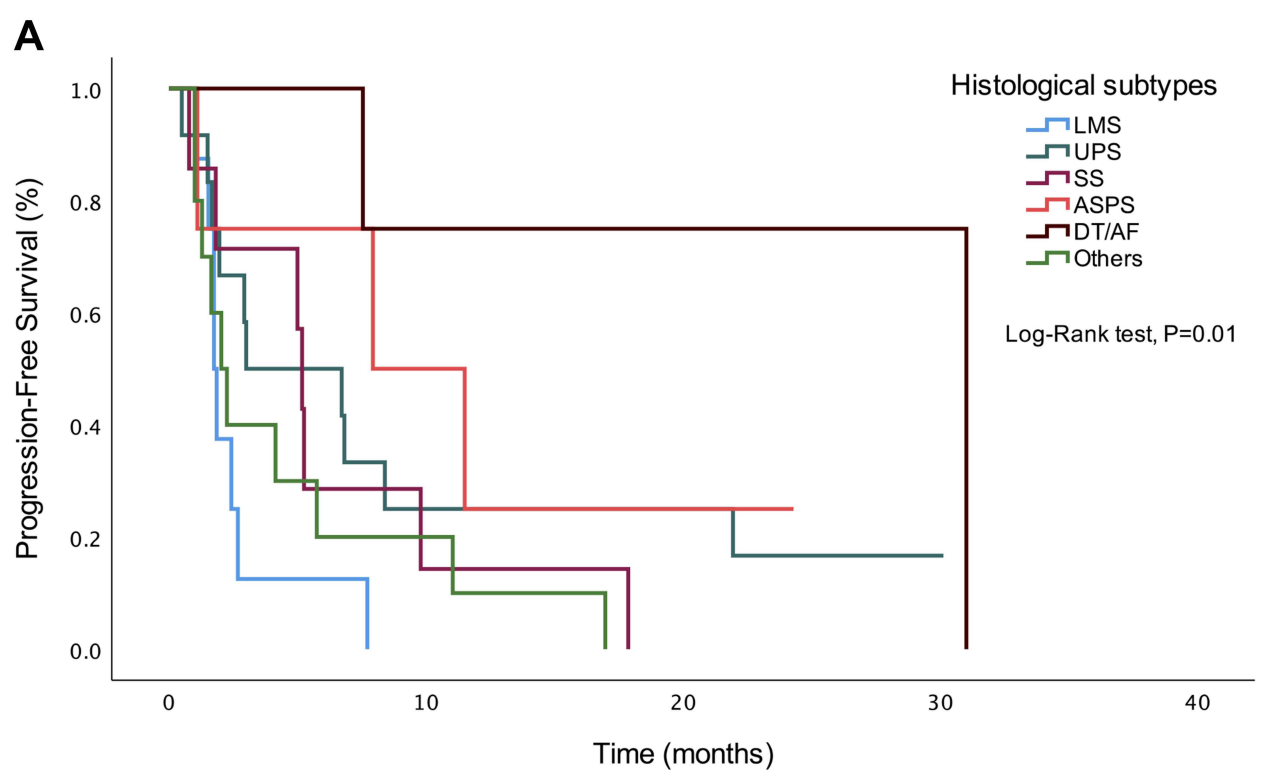

B

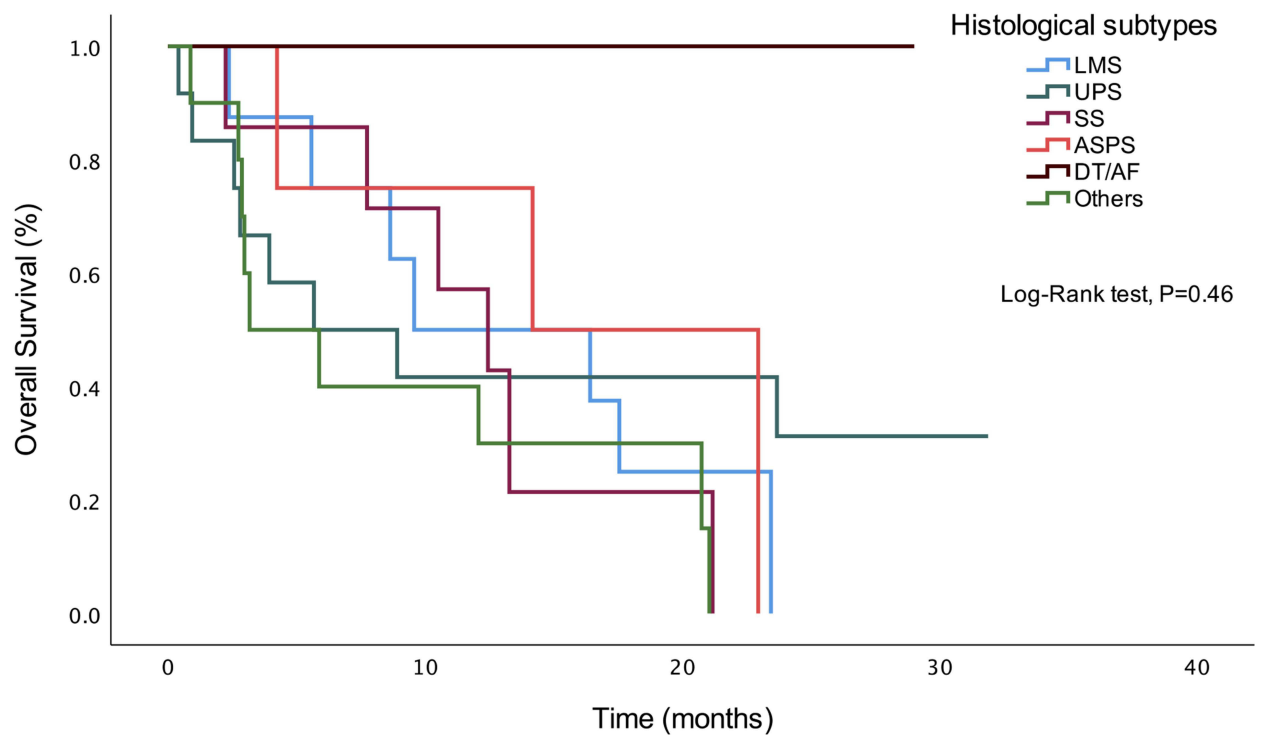

Figure 4 Kaplan-Meier curves for progression-free survival (A) and overall survival (B) of pazopanib based on histological subtypes of STS.

PALETTE trial, the DCR was $73 \%$ (6\% PR and $67 \%$ SD), the median PFS was 4.6 months, the OS was 12.5 months, and the median duration of treatment was 4.1 months. Table 4 summarizes the retrospective trials that assessed pazopanib for metastatic STS.

\section{Prognostic Factors}

Hypothyroidism has been found to be a prognostic factor for disease control. Moreover, hypothyroidism and response to pazopanib, better ECOG PS, histological subtypes DT/AF, and ASPS were favorable prognostic factors for PFS, and only the presence of hypothyroidism and response to pazopanib were the significant favorable variables for OS.

We showed that pazopanib-induced hypothyroidism was associated with better clinical outcomes, in agreement with the findings of Karaagac et al. However, Vos et al retrospectively assessed the combined results of the EORTC 62,043 and 62,072 trials and reported that there was no association between the occurrence of pazopanibinduced proteinuria, hypothyroidism, and cardiotoxicity and outcomes in patients with STS. ${ }^{30}$ In contrast, Bianchi 
Table 4 Summary of Pazopanib in Real-World STS Data

\begin{tabular}{|c|c|c|c|c|c|c|c|}
\hline Author/Year & Year/Region/No. Pts & $\begin{array}{l}\text { mAge } \\
\text { (Years) }\end{array}$ & $\begin{array}{l}\text { mDOT } \\
\text { (Months) }\end{array}$ & $\begin{array}{l}\text { ORR } \\
\text { (\%) }\end{array}$ & $\begin{array}{l}\text { DCR } \\
\text { (\%) }\end{array}$ & $\begin{array}{l}\text { mPFS } \\
\text { (Months) }\end{array}$ & $\begin{array}{l}\text { mOS } \\
\text { (Months) }\end{array}$ \\
\hline Karaagac et $\mathrm{al}^{27}$ & 2020/Turkish/79 & 49.6 & 6.1 & 16.5 & 45.6 & 3.97 & 11.4 \\
\hline Huang et al ${ }^{18}$ & 2018/Taiwanese/50 & 56.5 & - & 14 & 44 & 3.1 & 11 \\
\hline Nakamura et al $\left.\right|^{17}$ & 2016/Japanese//56 & 53.8 & 7.17 & 10.4 & 46.4 & 3.85 & 11.20 \\
\hline Koca et $\mathrm{al}^{26}$ & 2020/Turkish/ 103 & 50 & 3.8 & 20 & 42 & 4.3 & 10.1 \\
\hline Chung et $\mathrm{al}^{28}$ & 2020/Korean/ 347 & 51 & - & 15.6 & 54.8 & 5.3 & 12 \\
\hline Seto et $\mathrm{al}^{29}$ & 2019/California //23 & 60 & 3 & 10.5 & 38.2 & 3 & - \\
\hline Kollar et $\mathrm{a}^{25}$ & $\begin{array}{l}2017 / \text { EORTC phase II } \\
\text { and III }\end{array}$ & 52 & - & 46.9 & - & 3 & 9.9 \\
\hline Current study & 202I/Saudi/ 45 & 28 & 4.1 & 15.5 & 55.6 & 4.1 & 12.4 \\
\hline
\end{tabular}

et al reported that TKI-induced hypothyroidism might act as a potential biomarker for response and efficacy of treatment in patients with renal cell carcinoma. ${ }^{31}$ Furthermore, Bo et al recently reported that TKI-induced hypothyroidism is a prognostic factor for longer PFS in patients with metastatic renal cell cancer. ${ }^{32}$

The median time for pazopanib-induced hypothyroidism in our patients was 2 (IQR: 0.9-5.2) months; therefore, the fact that pazopanib-induced hypothyroidism was associated with longer PFS may be related to prolonged exposure to pazopanib. However, it may be useful to consider that the occurrence of drug-induced hypothyroidism may have prognostic value during pazopanib treatment in patients with STS.

The prognostic factors reported in previous studies are summarized in this section. In the PALLETE trial, lower tumor grade, better ECOG PS, and less systemic therapy line before pazopanib were associated with better PFS, and there were no statistical differences based on histological subtypes. However, the classification for analysis was LMS vs SA vs other sarcomas. ${ }^{14}$ Kasper et al analyzed patients who received pazopanib based on short and long PFS and OS from phase II and PALLETE trials, and they added normal hemoglobin before treatment to ECOG PS and tumor grade as a favorable factor for better survival outcome. However, the tumor grade was not significantly associated with PFS. ${ }^{9}$ Nakamura et al reported lower ECOG PS, female sex, and the number of systemic therapies before pazopanib and DoT as favorable factors for OS in Japanese patients. Furthermore, they found that patients with liposarcoma had worse PFS than those without LPS, and patients with ASPS had better PFS than those with other sarcomas. Interestingly, they compared patients who met the study criteria of the PALLETE trial with those who did not meet the criteria and found no statistical difference in PFS or OS. ${ }^{17}$ Chung et al reported that ECOG PS $(<2)$ and the number of systemic therapies before pazopanib $(<3)$ were significant prognostic factors for OS. In addition, they highlighted the variation in ORR (\%) and median PFS (months) in histological subtypes as follows: ASPS, $90 \%$, and 24.5; solitary fibrous tumor, $82.2 \%$ and 13 ; and SS, $66.7 \%$ and 5.5 ; LMS, $61.1 \%$ and 6; UPS, $59.6 \%$ and 5.5, respectively. ${ }^{28}$ Karaagac et al identified female sex, ECOG PF $(\leq 1)$, response to pazopanib, and hypothyroidism as favorable prognostic variables for DFS and OS in 26 patients. Huang et al reported that ECOG PS $(\leq 1)$ ( 0 or 1$)$ and developing hand-foot syndrome were favorable prognostic variables for PFS and OS in Taiwan patients. Moreover, anemia during pazopanib therapy, histological subtypes LMS vs SS vs ASPS were also significant for worse OS but not for DFS. ${ }^{18}$ Koca et al reported a multicenter analysis of Turkish patients and found no significant differences in PFS or OS based on histological subtypes of STS. Longer OS was associated with ECOG PS $(\leq 1)$ and tumor grade $(<3)$ Ki67 $(<20 \%)$; however, only ECOG PS remained a significant factor in the multivariate analysis. ${ }^{26}$

\section{Tolerability and Toxicity}

We found that the standard dose of $800 \mathrm{mg}$ pazopanib was well tolerated in our patients, and dose reduction was mandated in six patients, and three patients stopped 
pazopanib due to toxicities (fatigue, transaminitis, and Interstitial lung disease). The most common adverse effects were anemia (62\%), weight loss (53\%), hypertension $(51.1 \%)$, and hypothyroidism $(40 \%)$. In general, the adverse effects in our patients were within the reported toxicity profile of other studies with variations in frequencies as follows: in the PALETTE trial, the most common adverse effects were fatigue (65\%), diarrhea (58\%), nausea (54\%), weight loss (48\%), and hypertension (41\%). ${ }^{14}$

Nakamura et al reported that the most common adverse effects were hypertension (38\%), transaminitis (24\%), and diarrhea $(22 \%)$ in Japanese patients. Pazopanib was discontinued because of toxicity in $27 \%$ of cases, commonly due to liver disorders, fatigue, and pneumothorax. ${ }^{17}$ Karaagac et al reported that four patients (5.1\%) discontinued treatment due to toxicities (heart failure in one patient, hypertension, and transaminases in two patients). The most common adverse effects were anemia (49.4\%), hyperbilirubinemia $(40.5 \%)$, transaminitis $(35.4 \%)$, and thrombocytopenia (24.1\%). However, hypothyroidism was observed in $16.5 \%$ of patients. ${ }^{27}$ Huang et al reported that hypertension $(56 \%)$, skin toxicity (40\%), anemia $(38 \%)$, and transaminitis $(30 \%)$ were the most common toxic effects in their patients; they reported pneumothorax in 2 patients (4\%), a duodenal rupture in 1 patient, and tumor rupture in 1 patient each. ${ }^{18}$ Finally, Koca et al reported fatigue $(91 \%)$, anorexia $(74 \%)$, weight loss (64\%), hypertension (50\%), and pneumothorax in two patients. $^{26}$

Screening with echocardiography is not a standard course of care in our institution during pazopanib treatment unless there is an indication; however, there was no indication during therapy, and all patients who received anthracycline before pazopanib had a normal ejection fraction (EF). The PALETTE trial showed a decrease in EF of $11 \%$ vs $5 \%$ with placebo but with no fatality, whereas in our patients, this was not identified because we did not perform ECHO to assess EF as it is not a standard course of care. NLR has been suggested as an adverse prognostic biomarker for STS. ${ }^{33}$ However, pretreatment NLR was not associated with DCR, PFS, or OS in our study population.

We acknowledge that the small sample number of patients limits generalization; however, it is the first cohort from this region. Further studies are highly recommended for the prognostic variables of the clinical outcome of pazopanib in STS.

\section{Conclusions}

Pazopanib was found to be tolerable and effective, with outcomes comparable to those reported in clinical trials and realworld data. Hypothyroidism is a prognostic factor for disease control and is associated with prolonged PFS and OS. Further studies are recommended to validate these findings.

\section{Abbreviations}

CI, confidence interval; ECOG PS, Eastern Cooperative Oncology Group Performance Status; HFS, hand-foot syndrome; HR, hazard ratio; IQR, interquartile range; STS, Soft tissue sarcoma; OR, odds ratio; ORR, objective response rate; DCR, disease control rate; OS, overall survival; PFS, progression-free survival; UPS, Undifferentiated pleomorphic sarcoma; SS, Synovial sarcoma; LMS, Leiomyosarcoma; ASPS, Alveolar soft part sarcoma; DT/AF, Desmoid tumor/Aggressive fibromatosis.

\section{Data Sharing Statement}

All data generated or analyzed during this study are included in this published article.

\section{Funding}

There is no funding to report.

\section{Disclosure}

The authors report no conflicts of interest in this work. The authors declare that the research was conducted in the absence of any commercial or financial relationships that could be construed as a potential conflict of interest.

\section{References}

1. Burningham Z, Mia Hashibe LS. The epidemiology of sarcoma. Am $J$ Dis Child. 2012;2:14. doi:10.1186/2045-3329-2-14

2. Clark MA, Fisher C, Judson I, Thomas JM. Soft-tissue sarcomas in adults. $N$ Engl $J$ Med. 2005;353(7):701-711. doi:10.1056/ nejmra041866

3. American Cancer Society. Cancer facts \& figures; $2021 \mathrm{Am}$ Cancer Soc. 2021:1-70.

4. Stiller CA, Botta L, Brewster DH, et al. Survival of adults with cancers of bone or soft tissue in Europe-report from the EUROCARE-5 Study. Cancer Epidemiol. 2018;56(August):146-153. doi:10.1016/j. canep.2018.08.010

5. Fabiano S, Contiero P, Barigelletti G, et al. Epidemiology of soft tissue sarcoma and bone sarcoma in Italy: analysis of data from 15 population-based cancer registries. Sarcoma. 2020;2020:1-10. doi:10.1155/2020/6142613

6. Wafa AA. Kingdom of Saudi Arabia a Saudi health council national health information center. Saudi cancer registry cancer incidence report Saudi Arabia; 2016. Available from: https://nhic.gov.sa/en/ eServices/Documents/2016.pdf. 
7. Fletcher CDM, Bridge JA, Hogendoorn MF. World Health Organization Classification of Tumours of Soft Tissue and Bone. 4th ed. Lyon: IARC Press; 2013.

8. Gamboa AC, Gronchi A, Cardona K. Soft-tissue sarcoma in adults: an update on the current state of histiotype-specific management in an era of personalized medicine. CA Cancer J Clin. 2020;70 (3):200-229. doi:10.3322/caac.21605

9. Kasper B, Sleijfer S, Litière $S$, et al. Long-term responders and survivors on pazopanib for advanced soft tissue sarcomas: subanalysis of two European Organisation for Research and Treatment of Cancer (EORTC) clinical trials 62043 and 62072. Ann Oncol. 2014;25(3):719-724. doi:10.1093/annonc/mdt586

10. Seddon B, Strauss SJ, Whelan J, et al. Gemcitabine and docetaxel versus doxorubicin as first-line treatment in previously untreated advanced unresectable or metastatic soft-tissue sarcomas (GeDDiS): a randomised controlled Phase 3 trial. Lancet Oncol. 2017;18 (10):1397-1410. doi:10.1016/S1470-2045(17)30622-8

11. Judson I, Verweij J, Gelderblom H, et al. Doxorubicin alone versus intensified doxorubicin plus ifosfamide for first-line treatment of advanced or metastatic soft-tissue sarcoma: a randomised controlled phase 3 trial. Lancet Oncol. 2014;15(4):415-423. doi:10.1016/S14702045(14)70063-4

12. Karavasilis V, Seddon BM, Ashley S, Al-Muderis O, Fisher C, Judson I. Significant clinical benefit of first-line palliative chemotherapy in advanced soft-tissue sarcoma: retrospective analysis and identification of prognostic factors in 488 patients. Cancer. 2008;112 (7):1585-1591. doi:10.1002/cncr.23332

13. Blay JY, Van Glabbeke M, Verweij J, et al. Advanced soft-tissue sarcoma: a disease that is potentially curable for a subset of patients treated with chemotherapy. Eur J Cancer. 2003;39(1):64-69. doi:10.1016/S0959-8049(02)00480-X

14. Léon Bérard C, van der Graaf A, Chawla SP. Pazopanib for metastatic soft-tissue sarcoma (PALETTE): a randomised, double-blind, placebo-controlled phase 3 trial. Lancet. 2012;379(9829):1879-1886. doi:10.1016/S0140

15. Savina M, Le Cesne A, Blay JY, et al. Patterns of care and outcomes of patients with METAstatic soft tissue SARComa in a real-life setting: the METASARC Observational Study. BMC Med. 2017;15 (1):1-11. doi:10.1186/s12916-017-0831-7

16. Kitagawa D, Yokota K, Gouda M, et al. Activity-based kinase profiling of approved tyrosine kinase inhibitors. Genes Cells. 2013;18 (2):110-122. doi:10.1111/gtc.12022

17. Nakamura T, Matsumine A, Kawai A. The clinical outcome of pazopanib treatment in Japanese patients with relapsed soft tissue sarcoma: a Japanese Musculoskeletal Oncology Group (JMOG) Study. Cancer. 2016;122(9):1408-1416. doi:10.1002/cncr.29961

18. Huang PW, Chou WC, Shen WC, et al. Hand-foot skin reaction predicts treatment outcome of pazopanib in patients with metastatic soft tissue sarcoma: a multicenter study in the Asian population. Asia Pac J Clin Oncol. 2018;14(4):353-360. doi:10.1111/ajco.13029

19. Eisenhauer EA, Therasse P, Bogaerts J, et al. New response evaluation criteria in solid tumours: revised RECIST guideline (version 1.1). Eur $J$ Cancer. 2009;45(2):228-247. doi:10.1016/j. ejca.2008.10.026

20. Martin-Liberal J, Benson C, McCarty H, Thway K, Messiou C, Judson I. Pazopanib is an active treatment in desmoid tumour/aggressive fibromatosis. Clin Sarcoma Res. 2013;3(1):1-5. doi:10.1186/ 2045-3329-3-13
21. Jones RL, Katz D, Loggers ET, Davidson D, Rodler ET, Pollack SM. Clinical benefit of antiangiogenic therapy in advanced and metastatic chondrosarcoma. Med Oncol. 2017;34(10):1-5. doi:10.1007/s12032017-1030-2

22. Szucs Z, Messiou C, Wong HH, et al. Pazopanib, a promising option for the treatment of aggressive fibromatosis. Anticancer Drugs. 2017;28(4):421-426. doi:10.1097/CAD.0000000000000474

23. Toulmonde M, Pulido M, Ray-Coquard I, et al. Pazopanib or methotrexate-vinblastine combination chemotherapy in adult patients with progressive desmoid tumours (DESMOPAZ): a non-comparative, randomised, open-label, multicentre, Phase 2 Study. Lancet Oncol. 2019;20(9):1263-1272. doi:10.1016/S1470-2045(19)30276-1

24. Urakawa H, Kawai A, Goto T, et al. Phase II trial of pazopanib in patients with metastatic or unresectable chemoresistant sarcomas: a Japanese Musculoskeletal Oncology Group Study. Cancer Sci. 2020;111(9):3303-3312. doi:10.1111/cas.14542

25. Kollár A, Jones RL, Stacchiotti S, et al. Pazopanib in advanced vascular sarcomas: an EORTC Soft Tissue and Bone Sarcoma Group (STBSG) retrospective analysis. Acta Oncol (Madr). 2017;56 (1):88-92. doi:10.1080/0284186X.2016.1234068

26. Koca S, Beşiroğlu M, Özçelik M, et al. Pazopanib for metastatic soft-tissue sarcoma: a multicenter retrospective study. J Oncol Pharm Pract. 2020;27(3):541-6. doi:10.1177/1078155220924075

27. Karaağaç M, Sezgin Y, Eryılmaz MK, Araz M, Kaplan MA, Artaç M. The real-life outcome of pazopanib in patients with advanced soft tissue sarcoma: a Retrospective Cross-Sectional Study of a Turkish cohort. J Oncol Pharm Pract. 2020;26(7):1657-1666. doi:10.1177/ 1078155220904138

28. Chung OR, Hong JY, Kim JH, et al. Real-world outcomes of pazopanib treatment in Korean patients with advanced soft tissue sarcoma: a Multicenter Retrospective Cohort Study. Target Oncol. 2020;15(4):485-493. doi:10.1007/s11523-020-00731-z

29. Seto T, Song M-N, Trieu M, et al. Real-world experiences with pazopanib in patients with advanced soft tissue and bone sarcoma in Northern California. Med Sci. 2019;7(3):48. doi:10.3390/medsci7030048

30. Vos M, Sleijfer S, Litière S, et al. Association of pazopanib-induced toxicities with outcome of patients with advanced soft tissue sarcoma; a retrospective analysis based on the European Organisation for Research and Treatment of Cancer (EORTC) 62043 and 62072 clinical trials. Acta Oncol (Madr). 2019;58(6):872-879. doi:10.1080/ 0284186X.2019.1581376

31. Bianchi L, Rossi L, Tomao F, Papa A, Zoratto F, Tomao S. Thyroid dysfunction and tyrosine kinase inhibitors in renal cell carcinoma. Endocr Relat Cancer. 2013;20(5):R233-R245. doi:10.1530/ERC-130201

32. Bo L, Zhou L, Tang Y, Liu Y, Li H, Wang K. Tyrosine Kinase Inhibitors-Induced Hypothyroidism as a Prognostic Factor in Metastatic Renal Cell Carcinoma: a Systematic Review and MetaAnalysis. 2020:1-13[Preprint]. Available from: https://www. researchsquare.com/article/rs-36842/v1. doi:10.21203/rs.3.rs-36842/ v1

33. Liu G, Ke LC, Sun SR. Prognostic value of pretreatment neutrophil-to-lymphocyte ratio in patients with soft tissue sarcoma: a meta-analysis. Medicine (Baltimore). 2018;97(36):e121766. doi:10.1097/MD.0000000000012176 


\section{Publish your work in this journal}

Cancer Management and Research is an international, peer-reviewed open access journal focusing on cancer research and the optimal use of preventative and integrated treatment interventions to achieve improved outcomes, enhanced survival and quality of life for the cancer patient.

The manuscript management system is completely online and includes a very quick and fair peer-review system, which is all easy to use. Visit http://www.dovepress.com/testimonials.php to read real quotes from published authors.

Submit your manuscript here: https://www.dovepress.com/cancer-management-and-research-journa/ 